\title{
Leukocyte Esterase as Predictor of Urine Culture Result
}

Willis John Rieker ${ }^{1}$, Eileen Reilly ${ }^{2}$, Balamurugan Pandiyan ${ }^{3}$, Samantha Lom ${ }^{4}$ and Andrew Merry

${ }^{1}$ Department of Pathology and Medical Laboratory, Beloit Memorial Hospital, Wisconsin, USA

${ }^{2}$ Department of Health Science, SSM Health Janesville, Wisconsin, USA

${ }^{3}$ Department of Mathematics, University of Wisconsin, Wisconsin, USA

${ }^{4}$ Department of Medical Microbiology, Beloit Memorial Hospital, Wisconsin, USA

${ }^{5}$ Department of Academic Affairs, International University Health Science, Wisconsin, USA

\begin{abstract}
Objective: Assess varying levels of leukocyte esterase presence on urine dipstick as a risk factor for positive urine culture.

Materials and methods: Retrospective evaluation of outpatient laboratory data from Beloit Memorial Hospital obtained randomly from samples in outpatient settings in the year 2016. 2000 urine results obtained from automated urine dipstick and microscopy analysis. From the 2000 samples, 1123 patients randomly selected and grouped into controls and case participants based on positive urine cultures. Information gathered included age and gender.

Conclusion: Leukocyte esterase on dipstick analysis at "large" or "moderate" levels are both independent positive predictors of positive urine culture. "Small" level of leukocyte esterase has no predictive value for positive urine culture and "trace" leukocyte level has a negative predictive value for a positive urine culture.
\end{abstract}

Keywords: Leukocyte esterase; Microscopy analysis; Positive urine culture

\section{Introduction}

Urinary Tract infection (UTI) is a frequent outpatient diagnoses. It is common practice to obtain urine for dipstick and microscopy analysis for gastroenterology or genitourinary complaints. The challenge comes with interpretation since gold standard continues to be the delayed urine culture. Leukocyte esterase is an indicator on the urine dipstick analysis. Leukocyte esterase is an enzyme in neutrophils and macrophages that is released when those cells are injured. Based on Pappas article from 1991 "Leukocyte esterase may be used to detect $>10$ leukocytes per high power field (sensitivity of $75 \%$ to $96 \%$; specificity of $94 \%$ to $98 \%$ " [1]. How well does varying levels of leukocyte esterase equate to a positive urine culture which is the gold standard?

Another 2004 article by Deville' et al. reviewing the sensitivity and specificity of "BOTH nitrites and leukocyte esterase tests are positive then sensitivity (for bacterial UTI) $68 \%$ to $88 \%$ " [2]. This article combined the data rather than assessing leukocyte esterase separately. Once again, literature review reviewed sensitivity and specificity data of leukocyte esterase but in combination with presence of blood, not as a separate entity. "Nitrites OR leukocyte esterase AND blood had sensitivity and specificity 75 and 66\%" [3].

As stated by a meta-analysis of nitrites "overall the sensitivity of urine dipstick test for nitrites was low (45\% to $60 \%$ in most situations) with high levels of specificity ( $85 \%$ to $98 \%$ ) [2]. Nitrite positive dipstick allows provider to more confidently make the diagnosis of UTI. Unfortunately, nitrites generally only present if patient infected with Enterobacteriaceae organism that generates this by product by metabolism of nitrates. In order to detect nitrites in urine the patient cannot void for approximately 4 hours [4]. This is a problem when your patient is voiding less than every 4 hours which is common scenario in patient with suspected UTI.

If a medical provider reviews Medical textbook regarding the diagnostic testing in urinary tract infections (UTI) the following information is available. Harrison's Guide to Internal Medicine states "The leukocyte esterase dipstick method is less sensitive than microscopy in identifying pyuria but is useful alternative when microscopy is not feasible" [5]. Rosen's Emergency Medicine mentions "urine dipstick testing for leukocyte esterase has shown sensitivity $75 \%$ to $96 \%$ in detecting pyuria associated with urinary tract infection" [6] Merck Manual states "the leukocyte esterase is very specific for presence of $>10$ WBC's/microliter and is fairly sensitive [7]. "Epocrates": A urinary tract infection is indicated by urinalysis test result of "positive for leukocyte esterase, nitrites, and Hgb.”

Previous literature suggests that treatment in afebrile uncomplicated adult patient with no vaginal discharge with classic genitourinary symptoms can be treated without urinalysis. "In women who present with 1 or more symptoms of UTI the probability of infection is approximately $50 \%$. Specific combinations of symptoms (dysuria AND polyuria) raise the probability of UTI to more than $90 \%$ [8].

The problem is that patients will visit an outpatient setting for treatment with only one genitourinary complaint ("dysuria"), normal vitals and possibly another symptom such as mild suprapubic pressure. Often the initial assessment involves a urinalysis. A complete blood count may not be justifiable or immediately obtainable. Even more difficult is the urban or rural urgent care setting where urine microscopy may not be available. Often the urinalysis dipstick will indicate "small" or "trace" leukocytes and 5-10 WBC (if microscopy available). Previous literature has not looked at the varying levels of leukocyte esterase on the dipstick and the correlation of those levels with a positive urine culture. In the age of antibiotic resistance and thus

*Corresponding authors: Eileen Reilly, Department of Health Sciences SSM Health Janesville, Wisconsin, USA, Tel: 18774876012; E-mail: etreilly2004@yahoo.com

Received August 10, 2017; Accepted November 13, 2017; Published November 17, 2017

Citation: Rieker WJ, Reilly E, Pandiyan B, Lom S, Merry A (2017) Leukocyte Esterase as Predictor of Urine Culture Result. J Med Microb Diagn 6: 264 doi:10.4172/2161-0703.1000264

Copyright: (c) 2017 Rieker WJ, et al. This is an open-access article distributed under the terms of the Creative Commons Attribution License, which permits unrestricted use, distribution, and reproduction in any medium, provided the original author and source are credited. 
the judicious use of antibiotics, medical providers have to decide based on a marginal urinalysis result with "trace" or "mild" leukocyte esterase to treat the patient with antibiotics or wait for culture and then utilize more resources later as you contact the patient if the urine culture is positive. Just on the 1123 samples in this study, 442 sample were "mild" or "trace" which is $40 \%$ of the data.

\section{Materials and Methods}

Data base "Path Net" was accessed by Microbiology staff for patient urine laboratory information at Beloit Memorial Hospital. 2000 outpatient samples were randomly selected from each month of the year 2016. Urinalysis was initially completed by Siemens Atlas analyser. This laboratory equipment utilizes colorometric change for detection of leukocyte esterase. The degree of color change quantifies the classification of leukocyte esterase level. At the time this study was completed all urine samples at Beloit Memorial hospital are plated for growth. Leukocyte esterase levels are resulted as "large", "moderate", "small" and "trace". Other hospital and clinic labs may result leukocyte esterase in numerical values. According to the CLINITEX Novus Operations Guide [9].

\section{Large $=3+$; Moderate $=2+;$ Small $=1+$; and Trace $=$ Trace}

492 of the samples were positive for urine culture growth indicative of true UTI bacturia based on laboratory standards from_Cumitech $2 \mathrm{C}$ Laboratory Diagnosis of Urinary Tract Infections [10].

Utilizing accession numbers from laboratory data I was able to access patient medical record number to determine age and gender of the studied patient population (Figures 1 and 2).

\section{Results}

Odds ratio (OR) was utilized to determine association between

\section{Control group: age \& gender frequency}

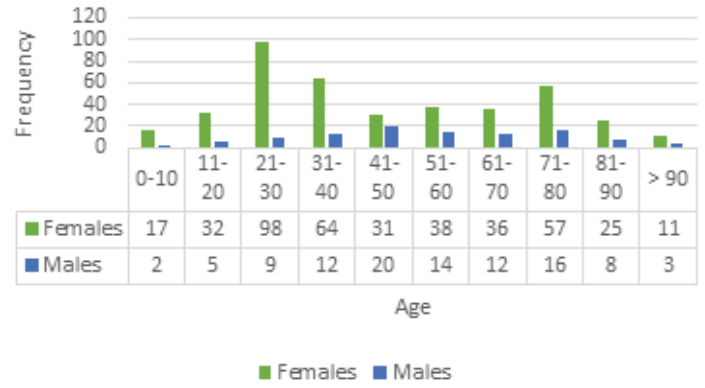

Figure 1: Control group: Age and gender frequency.

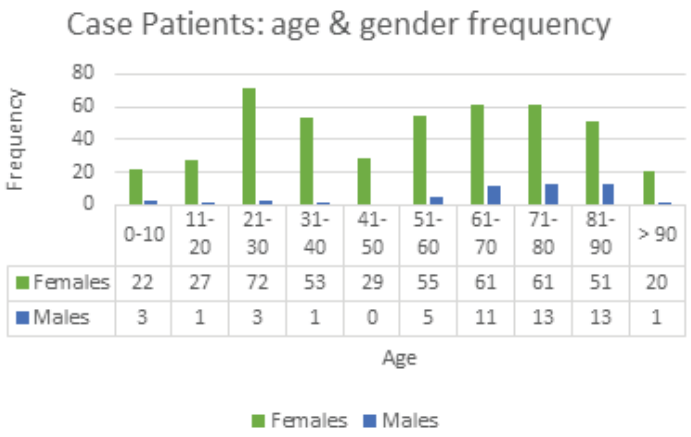

Figure 2: Case Patients: Age and gender frequency.

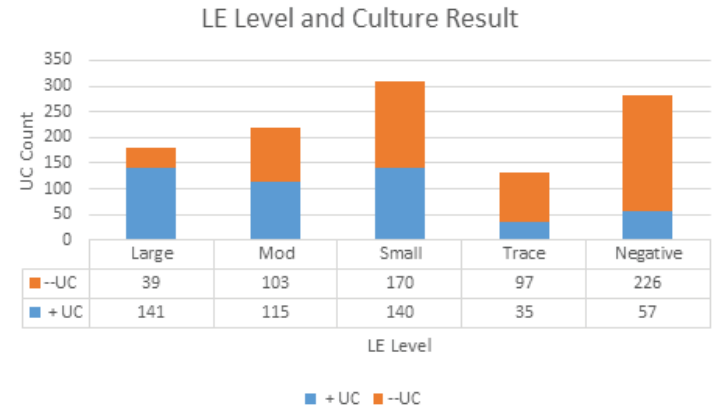

Figure 3: LE Level and culture result.

\begin{tabular}{|c|c|}
\hline \multicolumn{3}{|c|}{ “Large” (3+) LE 2 $\times \mathbf{2}$ table } \\
\hline 141 & 39 \\
\hline 347 & 596 \\
\hline
\end{tabular}

$\mathrm{OR}=6.21$

Confidence Interval (CI) 4.25-9.07

p-value: $2.5 \times 10^{-5}<0.05$

$\mathrm{H}_{0}$ : OR>1, so "Large" LE has strong association with Positive Urine culture (+UC)

Table 1: Large leukocyte esterase.

\begin{tabular}{|c|c|}
\hline \multicolumn{3}{|c|}{ “Moderate” (2+) LE 2 × 2 table } \\
\hline 115 & 103 \\
\hline 373 & 532 \\
\hline
\end{tabular}

$\mathrm{OR}=1.59$

Confidence Interval $(\mathrm{Cl}) 1.18-2.14$

p-value: $0.0014<0.05$

$\mathrm{H}_{0}$ : OR>1, so "Moderate" LE has strong association with Positive Urine culture (+UC)

Table 2: Moderate leukocyte esterase.

\begin{tabular}{|l|c|}
\hline \multicolumn{2}{|c|}{ "Small” (1+) LE 2 $\times 2$ table } \\
\hline \multicolumn{1}{|c|}{140} & 170 \\
\hline \multicolumn{1}{|c|}{348} & 465 \\
\hline $\begin{array}{l}\text { OR=1.10 } \\
\text { Confidence Interval (Cl) } 0.84-1.43 \\
\text { p-value: } 0.2592>0.05 \\
H_{0}: \text { OR=1.1, so "Small" LE has no association with Positive Urine culture (+UC) }\end{array}$ \\
\hline
\end{tabular}

Table 3: Small leukocyte esterase.

\begin{tabular}{|l|c|}
\hline \multicolumn{2}{|c|}{ "Trace" LE 2 $\times 2$ table } \\
\hline \multicolumn{1}{|c|}{35} & 97 \\
\hline \multicolumn{1}{|c|}{453} & 538 \\
\hline $\begin{array}{l}\text { OR=0.43 } \\
\text { Confidence Interval }(\mathrm{Cl}) 0.29-0.65 \\
\text { p-value: } 0.000015627<0.05 \\
\mathrm{H}_{0}: \text { OR<1, so "Trace" LE has strong negative association with Positive Urine } \\
\text { culture (+UC) }\end{array}$ \\
\hline
\end{tabular}

Table 4: Trace leukocyte esterase.

Leukocyte esterase and positive urine culture given case control study. The data for the $2 \times 2$ frequency table was obtained from Figure 3. Formula $\mathrm{ad} / \mathrm{bc}$ for odds ratio [11]. $2 \times 2$ data and $O R$ values then applied to mathematical formula for Confidence interval: $e^{\wedge}[\ln (O R)+/-1.96 \sqrt{1 / a+1 / b+1 / c+1 / d]}$ With 5\% level of significance, the $\mathrm{p}$-value has been determined utilizing Fisher exact test (Tables 1 and 2).

For all categories, "Large", "Moderate", "Small" and "Trace" Leukocyte Esterase, we define the null hypothesis $\left(\mathrm{H}_{0}: \mathrm{OR}=1\right)$, that is to say, Leukocyte Esterase is not associated with the positive urine culture (Tables 3 and 4). 


\section{Discussion}

Leukocyte esterase has a strong predictive value of positive urine culture at "moderate" or "large" levels based on statistical hypothesis testing of odds ratio. Leukocyte esterase at "small" levels has no predictive value for association with positive urine culture thus accepting null hypothesis. "Trace" leukocyte esterase has NEGATIVE predictive value for association with positive urine culture therefore rejecting null hypothesis.

Out of 1123 urine samples only 398 were positive for "large" or "moderate" LE and 442 sample were "small" or "trace". Determining association for all levels of LE is pertinent given that the majority of urine samples in this data set are not "large" or "moderate" and more closely resembles the distribution in the typical outpatient setting. Applying statistical analysis to the LE levels gives the provider more confidence in opting for medicinal intervention or not.

\section{Conclusion}

Specifically, the results can be applied in outpatient clinics where microscopy is not readily available, and symptoms are vague. Possibly the information may help to curb unnecessary antibiotic prescriptions. The data can also support the reduction of additional processing of select urine samples sent to laboratories for further evaluation. This reduction of higher level of testing will ultimately decrease health care costs for the patient and the clinic.

\section{References}

1. Pappas PG (1991) Laboratory in the diagnosis and management of urinary tract infections. Med Clin North Am 75: 313.

2. Deville WL, Yzermans JC, Van Duijn NP, Bezemer PD, Van Der Windt DAWM et al. (2004) The urine dipstick test useful to rule out infections. A meta-analysis of the accuracy. BMC Urol 4: 4

3. Little P, Turner S, Rumsby K, Jones R, Warner G, et al. (2010) Validating the prediction of lower urinary tract infection in primary care: Sensitivity and specificity of urinary dipsticks and clinical scores in women. $\mathrm{Br} J$ Gen Pract 60:495.

4. Williams GJ, Macaskill P, Chan SF, Turner RM, Hodson E, et al. (2010) Absolute and relative accuracy of rapid urine tests for urinary tract infection in children: $A$ meta-analysis. Lancet Infect Dis 10: 240.

5. Dennis MD (2005) Renal and urinary tract function. Harrison's Guide to Internal Medicine. (16th edn) 8: 1718

6. John MD (2010) Genitourinary System. Rosen's Emergency Medicine. (6th edn) 44:1299.

7. Mark MD (2006) Genitourinary Disorders. Merck Manual. (18th edn) 17: 1971

8. Bent S, Nallamothu BK, Simel DL, Finn SD, Saint S (2002) Does this woman have an acute uncomplicated urinary tract infection? JAMA 287:2701.

9. http://a360-wp-uploads.s3.amazonaws.com/wp-content/uploads clpmag/2015/08/141229-UC1_CLINITEK_Novus_v1.1_US_WP.FINAL2_.pdf

10. McCarter $Y$ (2009) Laboratory diagnosis of urinary tract infections. Cumulative Techniques and Procedures in Clinical Microbiology 2C.

11. Szumilas M (2010) Explaining odds ratio. J Can Acad Child Adolesc Psychiatry 19: $227-229$ 